\title{
PIEZOELECTRIC TUNER COMPENSATION OF LORENTZ DETUNING IN SUPERCONDUCTING CAVITIES *
}

\author{
G. K. Davis, J. R. Delayen ${ }^{\#}$ \\ Thomas Jefferson National Accelerator Facility, Newport News, VA 23606
}

\begin{abstract}
Pulsed operation of superconducting cavities can induce large variations of the resonant frequency through excitation of the mechanical modes by the radiation pressure. The phase and amplitude control system must be able to accommodate this frequency variation; this can be accomplished by increasing the capability of the rf power source. Alternatively, a piezo electric tuner can be activated at the same repetition rate as the rf to counteract the effect of the radiation pressure. We have demonstrated such a system on the prototype medium beta SNS cryomodule [1] with a reduction of the dynamic Lorentz detuning during the rf pulse by a factor of 3 . We have also measured the amplitude and phase of the transfer function of the piezo control system (from input voltage to cavity frequency) up to several $\mathrm{kHz}$ [2].
\end{abstract}

\section{DYNAMIC LORENTZ DETUNING}

SNS will be the first large-scale use of superconducting cavities in a pulsed accelerator. Although the cavities will be strongly beam-loaded the dynamic Lorentz detuning is expected to be a substantial fraction of the bandwidth and the phase and amplitude control under these conditions will require additional amounts of rf power. During tests of the SNS prototype cryomodule [1,2], the dynamic behavior of the frequencies of the 3 cavities was measured and the use of a piezo tuner for its compensation was evaluated.

The measurements were made with a cavity resonance monitor (CRM) [3]. The CRM has a $50 \mathrm{~dB}$ dynamic range, so it can measure the cavity frequency during the rise and decay of the fields, but we do not have, at present, the ability to measure the frequency between pulses. This would require maintaining a small but finite rf field in the cavity.

A typical measurement of the dynamic Lorentz detuning for an "SNS" pulse is shown in Fig. 1. In that figure, and all similar ones, the transients at the beginning and the end are associated with the phase-lock loop acquiring and losing lock and are not significant.

\section{PIEZO TUNER TRANSFER FUNCTIONS}

The transfer function (phase and amplitude) from input voltage of the amplifier driving the piezo to cavity frequency was measured. This was done by sweeping the frequency of the drive modulation, and measuring

\footnotetext{
* Work supported by the U.S. Department of Energy under contract DE-AC05-00-OR22725

\#delayen@jlab.org
}

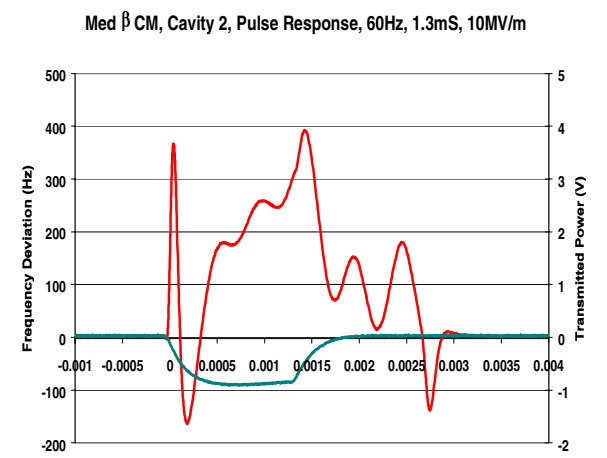

Figure 1: Dynamic Lorentz detuning during pulsed operation

simultaneously the phase and amplitude of the frequency modulation.

For cavity 2 we measured the transfer function for the two extreme positions of the slow mechanical tuner (Fig. 2). Subtle but real differences in the responses were observed

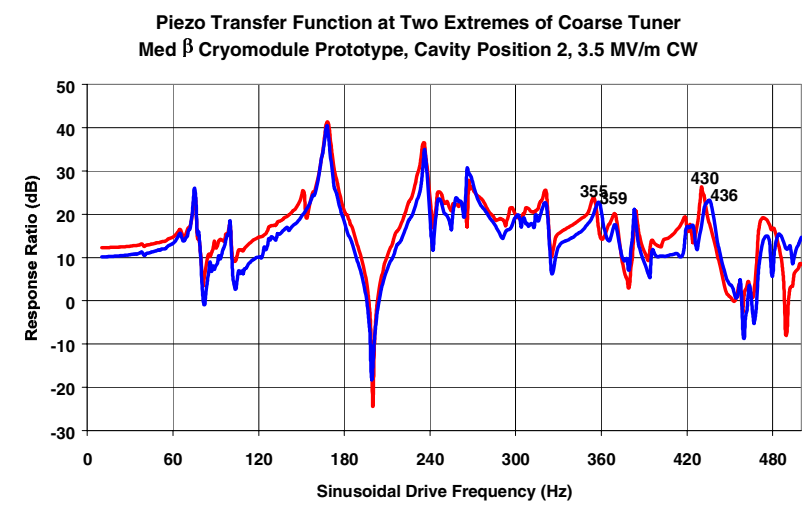

Figure 2: Amplitude of the piezo transfer function in cavity 2 for the two extreme positions of the coarse mechanical tuner. Blue: $804.612 \mathrm{MHz}$; Red: $805.06 \mathrm{MHz}$

The behavior of the piezo tuner transfer function was somewhat unexpected and is not, at present, fully understood. First, the transfer functions of the 3 cavities were substantially different one from the other; some strong resonances that were present in one cavity were absent in others (Fig. 3). Most surprising was the large amount of phase shift as the modulation frequency was increased; more than $20 \pi$ had accumulated at $500 \mathrm{~Hz}$. It should be pointed out that the transfer function includes the driver amplifier for the piezo, the piezo itself, and the 
mechanical modes of the cavity. Separation of the various contributions will require further testing.

Cavity Pos 1, Piezo Transfer Function
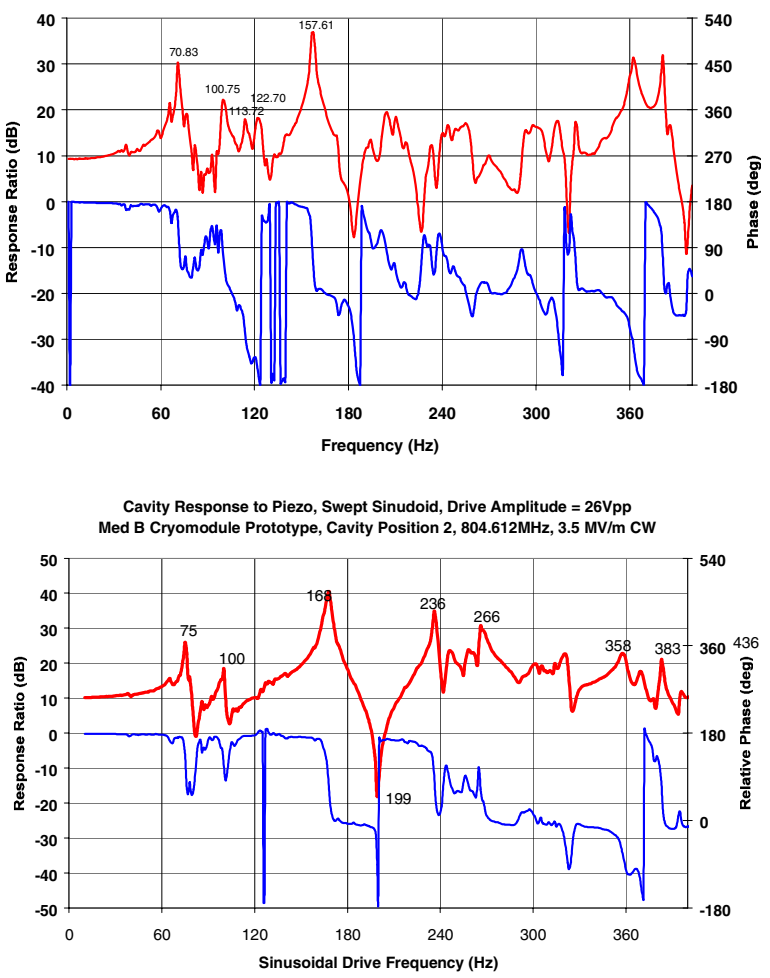

Cavity Position 3, Piezo Transfer Function

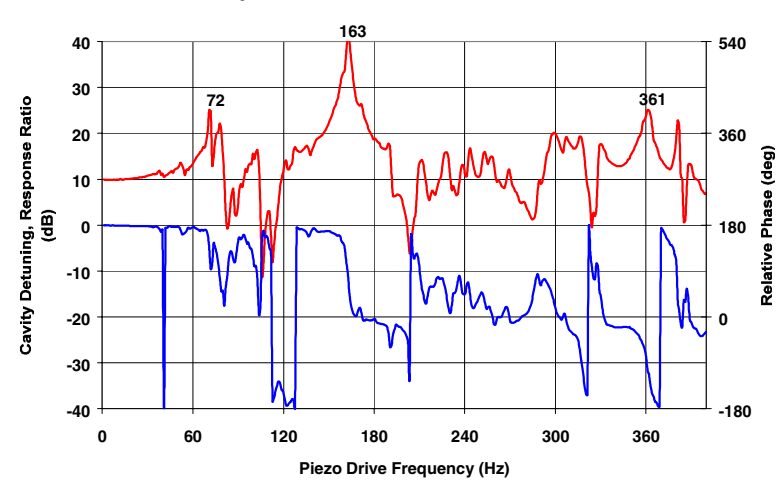

Figure 3: Phase and amplitude of the piezo transfer functions for the 3 cavities.

A complete transfer function to $3.2 \mathrm{kHz}$ was measured for cavity 3 (Fig. 4). Note the continuous accumulation of phase shift and the broad peak around $2 \mathrm{kHz}$.

\section{PULSED OPERATION OF THE PIEZO TUNER}

The length of the rf and piezo pulses are much shorter than the period of the dominant mechanical modes so it would be expected that the detail of the pulse shape would have relatively little effect on the dynamic behavior of the cavity frequency. Furthermore the decay time of the dominant modes is much larger than the spacing between the pulses, thus it would be expected that the cavities would be in a perpetual state of "ringing. This is
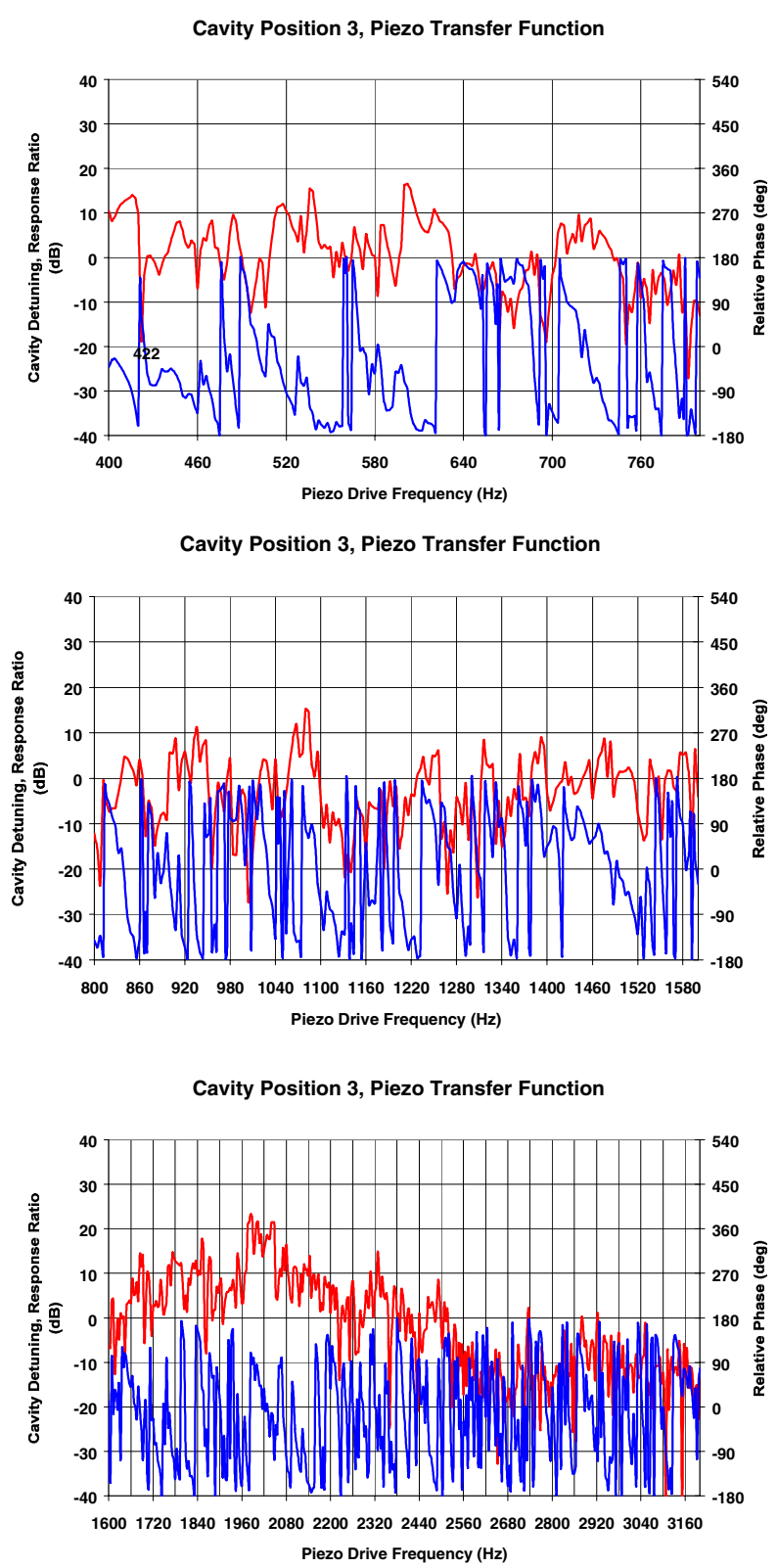

Figure 4: Amplitude and phase of the piezo transfer function for cavity 3 from 400 to $3200 \mathrm{~Hz}$. Results up to $400 \mathrm{~Hz}$ are shown in Fig. 3.

demonstrated in the following Fig. 5. Cavity 2 was operated cw and the piezo tuner was activated at $60 \mathrm{~Hz}$, in a similar fashion and amplitude that would be needed to compensate for the Lorentz detuning. The response of the cavity frequency was relatively insensitive to the rise time of the piezo pulse, but it was also essentially periodic. It is for this reason that, for the short duration of the $\mathrm{rf}$ pulse, the piezo pulse can be used to compensate for the Lorentz detuning which would have a similar complex, but periodic, behavior by carefully adjusting the timing between the two. 
Cavity Position 2 Response to Piezo Pulses at $1.28 \mathrm{~ms}$ pulse width; 160 usec rise time; $60 \mathrm{~Hz}$

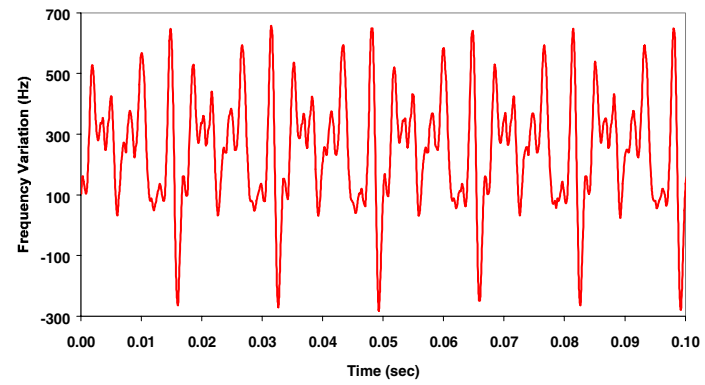

Figure 5: Cavity frequency variation induced by a 60 $\mathrm{Hz}$ excitation of the piezo tuner similar to that which would be needed to compensate for the rf-induced Lorentz detuning.

Of concern for use of the piezo tuner to control microphonics is the amount of microphonics that it can generate during activation. The frequency of cavity 2 was measured while activating the piezo tuner with a slow (1 or $2 \mathrm{~Hz}$ ) trapezoidal signal with rise time of $0,5,10$, and 20 msec. A square wave ( 0 rise time) generates microphonics of the same order of magnitude as the steady state displacement it induces; these microphonics then decay in about $400 \mathrm{msec}$ (Fig. 6). In order for the microphonics to be less than $50 \%$ of the steady state displacement, the ramp time of the trapezoidal drive must be at least 5 msec.

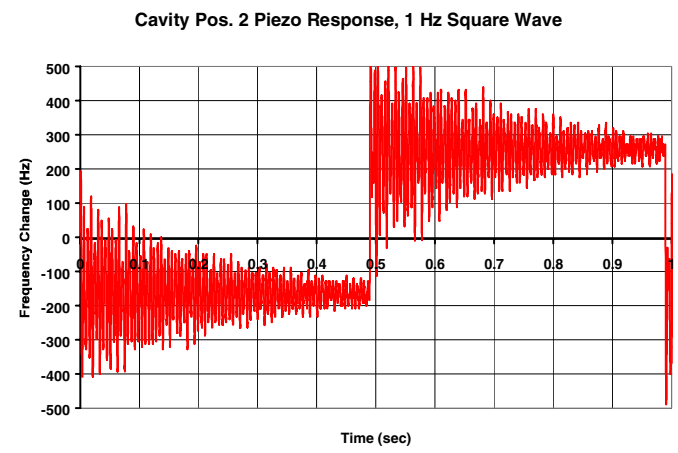

Figure 6: Cavity frequency response to square-wave excitation of the piezo tuner.

\section{PIEZO TUNER COMPENSATION OF DYNAMIC LORENTZ DETUNING}

The effectiveness of the piezo tuners in compensating for the Lorentz detuning is shown in Fig.7. A reduction by a factor of 3 was easily achieved. The critical parameter was the timing between the rf pulse and the piezo pulse. Similar reduction was achieved by changing the polarity of the piezo drive signal by adjusting the timing. It should be noted that this compensation takes place only during the short rf pulse. Although we do not have the ability to measure it, it is likely that the cavity frequency undergoes large transients between the rf pulses.

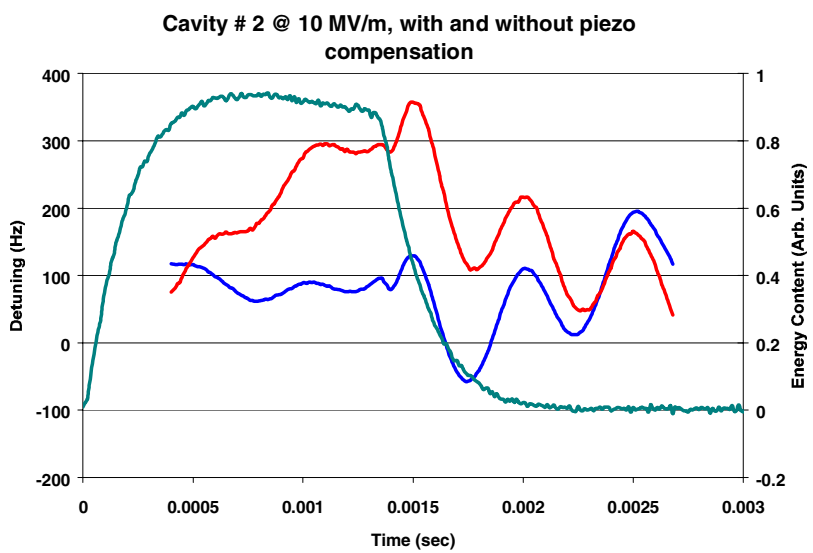

Figure 7: Cavity frequency without (red) and with (blue) piezo tuner compensation. Green: energy content

\section{DISCUSSION}

We have demonstrated a reduction by more than a factor of 3 of the dynamic Lorentz detuning of the SNS medium- $\beta$ prototype cryomodule during pulsed operation. While this more than exceeds the SNS requirements further reduction is possible with better optimization.

The transfer function between piezo actuation and cavity frequency revealed a complex behavior that varied from cavity to cavity. Large-scale use of piezo tuners for control of microphonics during $\mathrm{cw}$ operation will have to include sufficient adaptability and flexibility to accommodate this variability.

\section{REFERENCES}

[1] Jean Delayen, Ed Daly, Kirk Davis, Steve Smee, "Frequency Measurements on the Prototype SNS Medium- $\beta$ Cryomodule Under Pulsed and CW Operation," JLab Tech Note 02-049.

[2] I. Campisi, G. Ciovati, G. K. Davis, J. R. Delayen, M. Drury, L. W. Funk, J. Mammosser, T. Powers, J. Preble, C. E. Reece, C. H. Rode, M. Stirbet, H. Wang, T. Witlatch, K. M. Wilson, M. Wiseman, "The SNS Prototype Cryomodule: Testing and Performance," these proceedings RPAB065.

[3] G. Kirk Davis, Jean Delayen, Michael Drury, Thomas Hiatt, Curt Hovater, Thomas Powers, Joseph Preble, "Microphonics Testing of the CEBAF Upgrade 7-Cell Cavity," PAC 2001, Chicago, IL, 1822 June 2001. 